\title{
FIRST RESULTS OF QUASI-ISOCHRONOUS OPERATION AT LNLS
}

\author{
$\underline{\text { Liu Lin }}^{*}$ and P.F. Tavares, LNLS, Campinas, Brazil
}

\begin{abstract}
We have started a program at LNLS to implement a quasi-isochronous operation mode at the $1.37 \mathrm{GeV}$ electron storage ring. The aim is to explore non-linear longitudinal beam dynamics along with the reduction in the electron bunch length. To characterize the modes we have set up an electron bunch length measurement system which uses the visible light emitted from a bending magnet and a commercial fast photodiode with a sampling oscilloscope. The synchrotron oscillation frequency and the orbit displacement were also measured as a mean to assess the momentum compaction factor $\alpha$. Up until the moment a reduction of a factor of 13 in $\alpha$ was obtained.
\end{abstract}

\section{INTRODUCTION}

We describe the first experiments performed at the LNLS synchrotron light source where the momentum compaction factor is reduced in order to reduce the electron bunch length. Similar experiments were carried out at other laboratories [1]. The equilibrium bunch length in a storage ring is proportional to the square root of the linear momentum compaction factor, $\alpha_{1}$. Since $\alpha_{1}$ is proportional to the integral of the dispersion function in the dipoles, we can reduce it by focusing the dispersion in the dipoles so that a negative contribution to the integral is created canceling out part of the positive contribution. In principle, by shaping the dispersion function through the dipoles, the momentum compaction factor can be made as small as desired and even negative. In practice, however, several problems related to higher order longitudinal resonances and beam instabilities limit the value of $\alpha_{1}$ and require control on other parameters such as higher order terms of the momentum compaction factor and rf parameters. We present in this report the preliminary results of our efforts to operate the LNLS UVX electron storage ring in a quasi-isochronous (QI) configuration.

\section{THE QI OPTICAL CONFIGURATION AND MIGRATION FROM STANDARD OPTICS}

The LNLS storage ring lattice is composed of six doublebend arcs connected by six long straight sections. The lattice is flexible enough to be tuned to various optical configurations. In the standard configuration, which is used both for regular user operation and for injection, the arcs are made achromatic, which leaves the long straight sections dispersion free. In this case the dispersion function is always positive and corresponds to a momentum compaction factor of $8.3 \times 10^{-3}$. To reduce this value, we overfocus the dispersion function at the dispersive sections creating a negative contribution to the dispersion integral in the dipoles. A six fold symmetric quasi-isochronous model, QI6, is calculated for UVX in which the linear momentum compaction factor is reduced by a factor of 100 with respect to the standard mode. The bunch length should then be reduced by a factor of 10 , changing from approximately $40 \mathrm{ps}$ rms to $4 \mathrm{ps} \mathrm{rms}$.

The betatron tunes were adjusted in the QI6 mode to the same value as the standard mode. In this way, it is possible to transfer the optical configuration from the standard mode to the quasi-isochronous mode with the tunes fixed along the whole migration route. This operation scheme avoids the difficulties related to setting up injection conditions in the quasi-isochronous mode and also allows the opportunity to observe the beam behavior as the momentum compaction is slowly reduced.

We define the parameter $\chi$ as the ratio of the dispersion function at the BPMs in the long straight section to the dispersion function at the BPMs in the short section, as shown in Figure 1:

$$
\chi=\frac{\eta_{\text {BPM_Long }}}{\eta_{\text {BPM_Short }}}
$$

This parameter can be used to characterize the isochronicity of a configuration since the momentum compaction factor is uniquely determined by the value of the dispersion function at these BPMs for a 6-fold symmetric mode in UVX. As defined above, $\chi$ depends only on the orbit change at the BPMs for a given variation in the $\mathrm{rf}$ frequency. We have established a migration route with 18 intermediate configurations were $\chi$ varies monotonically from zero in the standard mode to -0.78 in the QI6 mode (Figure 2). Figure 3 shows $\alpha_{1}$ and the synchrotron oscillation frequency along the migration route.

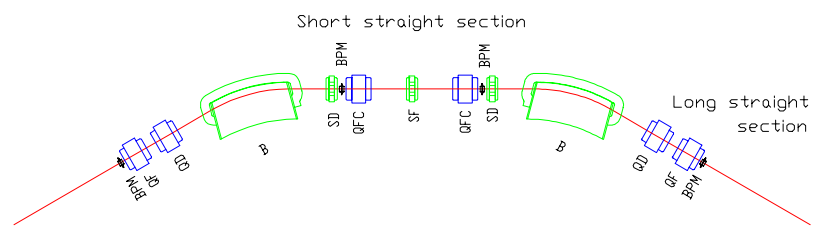

Figure 1: One basic cell of the UVX storage ring. 


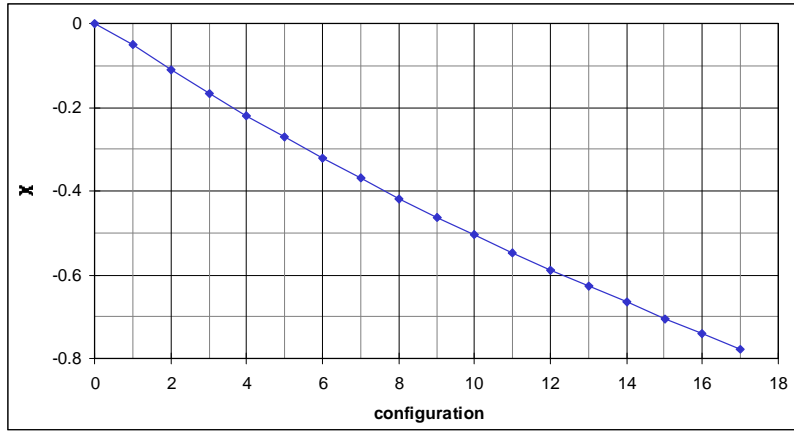

Figure 2: Calculated $\chi$ for the configurations in the migration route from the standard mode to the QI6 mode.

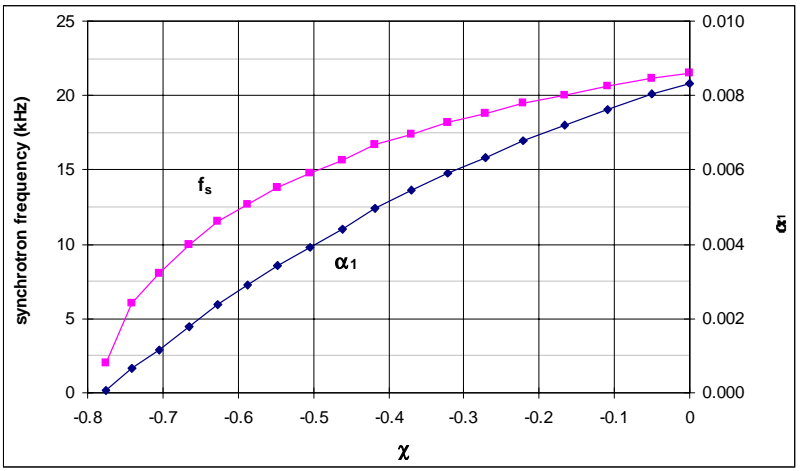

Figure 3: Calculated $\alpha_{1}$ and synchrotron oscillation frequency (for $\mathrm{V}_{\mathrm{rf}}=340 \mathrm{kV}$ ) as a function of $\chi$.

\section{EXPERIMENTAL RESULTS}

The experiments were carried out at the electron energy of $1.37 \mathrm{GeV}$ with a few bunches filled (15 to 25 instead of the usual 100) to minimize longitudinal coupled bunch effects. The number of filled bunches cannot be controlled very precisely with the present hardware. An upgrade for the injection system is being developed to allow for operation with programmed filling patterns.

The electron beam is injected into the storage ring at $120 \mathrm{MeV}$ and ramped to the standard optical configuration at $1.37 \mathrm{GeV}$. The 18 configurations of the migration route are then implemented one by one. In the first attempts, the tunes and the orbits had to be corrected at each step. As the momentum compaction is reduced, beam stability becomes more critical: orbit correction becomes more and more difficult, variations in parameters such as the rf frequency or the betatron tunes can cause larger changes in the beam lifetime and cause greater current drops. Up until the moment we have succeed to implement up to the 17th configuration (E17) in the migration route. To characterize the modes, we have measured the bunch length, the synchrotron frequency and the horizontal orbit displacement as a function of the rf frequency.

\section{$3.1 \chi$ Measurement}

We have measured the isochronicity parameter $\chi$ for some configurations in the migration route from the standard to the QI6 mode. For a given change in the rf frequency, the isochronicity parameter is given by $\chi=\Delta \mathrm{x}_{\text {BPM_Long }} / \Delta \mathrm{x}_{\text {BPM_Short }}$. Since there is a spread in the measured orbit displacements at the BPMs of the same group, we take the average displacement in each group. The results show that we have reduced $\chi$ to -0.74 . This value corresponds to a theoretical momentum compaction factor of $6.5 \times 10^{-4}$, a factor of 12.8 smaller than the value for the standard mode. In Figure 4 we show the measured synchrotron tune as a function of the measured parameter $\chi$ as well as the theoretical curve.

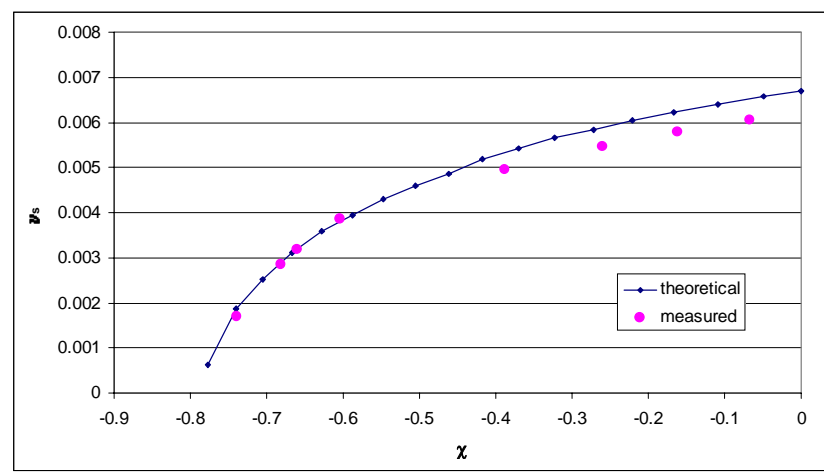

Figure 4: Theoretical and measured values of the synchrotron tune as a function of the isochronicity parameter $\chi$ during migration from the standard to the QI6 mode.

\subsection{Synchrotron Oscillation Frequency Measurement}

If the rf gap voltage is maintained at a constant value, changes in optical configurations can only modify the momentum compaction factor. We can thus derive the reduction in $\alpha$ in going from the standard mode to the QI6 mode from the measured synchrotron oscillation frequency. The measured values are $19.5 \mathrm{kHz}$ and 5.5 $\mathrm{kHz}$ respectively for the standard and QI6 modes for $\mathrm{V}_{\text {gap }}=316 \mathrm{kV}$. This corresponds to a reduction of a factor of 12.6 in $\alpha$ and agrees with the value measured in the previous section.

\subsection{Bunch-Length Measurement}

The bunch length is measured by an optical system that couples the visible light emitted by the electron bunch into an ultra-fast photodetector (7 ps FWHM). The signal is captured by a $20 \mathrm{GHz}$ sampling oscilloscope triggered by the zero-bunch signal generated from the storage ring master clock. The bunch length is obtained by fitting a gaussian to the data.

To estimate the time resolution of the system we have measured the bunch length as a function of the rf gap voltage in the standard operation mode with a few bunch configuration. The measured bunch length is about twice the expected value from theoretical calculations. Qualitatively, the expected increase in bunch length (and 
decrease in synchrotron oscillation frequency) produced by the reduction of the rf gap voltage is observed. The apparent very poor time resolution of this measurement system may have many contributions, which can be separated essentially into two types: (i) intrinsic resolution of the measurement system and (ii) coherent longitudinal oscillations of the bunches. The intrinsic resolution of the measurement system can be affected by the jitter of the trigger signal, the frequency bandpass of the measuring equipment and imperfections in cables and connectors. The jitter of trigger signal has been reduced to $2.5 \mathrm{ps} \mathrm{rms}$ and is thus negligible but the overall bandwidth of the system may be limited in our system. One indication is the fact that we have observed a narrow harmonic composition of the fast photodiode signal on a spectrum analyser. Nevertheless, the intrinsic overall resolution of the system could be subtracted from the measured bunch length data (if we assume that this is given by the quadratic sum of the actual bunch length and the resolution of the system). The other kind of contribution to the enlargement of the measured bunch duration, the coherent longitudinal oscillation of the beam, is more difficult to eliminate and there are indications that it occurred during the measurements. One evidence is the correlation between bunch lengthening and the increase in the observed number and intensity of the synchrotron frequency harmonics. We also observed a trembling of the beam image taken from a dispersive point in the dipole. Furthermore, the operation with few bunches is essential to get repetitive results. The bunch length measured when we operate in the 100 filled bunches mode has a large noise. The contribution of beam longitudinal oscillations to the measured length depends on many parameters and cannot be subtracted from the measured value. In this case, changes in the hardware must be implemented to overcome the limitations. One solution is to use a technique to measure the bunch in a single pass (e.g., streak camera, autocorrelation). Another solution is to implement the single bunch operation mode with a trigger signal generated by the bunch itself. Yet another solution is to eliminate the longitudinal oscillations via a feedback system. All these solutions require a substantial effort (technical and/or financial) to be implemented and we are presently analysing the various alternatives. The single bunch operation and a streak camera may be available in a near future. The present system, however, can be used to observe the qualitative behavior of the bunch length in these configurations.

In Figure 5 we show the measured bunch length (one sigma) as a function of the synchrotron oscillation frequency during migration from the standard mode $\left(\mathrm{f}_{\mathrm{s}}=20 \mathrm{kHz}\right)$ to the mode E17 $\left(\mathrm{f}_{\mathrm{s}}=6 \mathrm{kHz}\right)$. Initially the measured bunch length and the synchrotron frequency decrease with the reduction of the momentum compaction factor. From the configuration which corresponds to $\mathrm{f}_{\mathrm{s}}=8.5 \mathrm{kHz}$ on, further reduction of the momentum compaction factor still decreases the synchrotron frequency but not the measured bunch length, which starts fluctuating at larger values. This may be caused by the excitation of longitudinal instabilities in low alpha configurations.

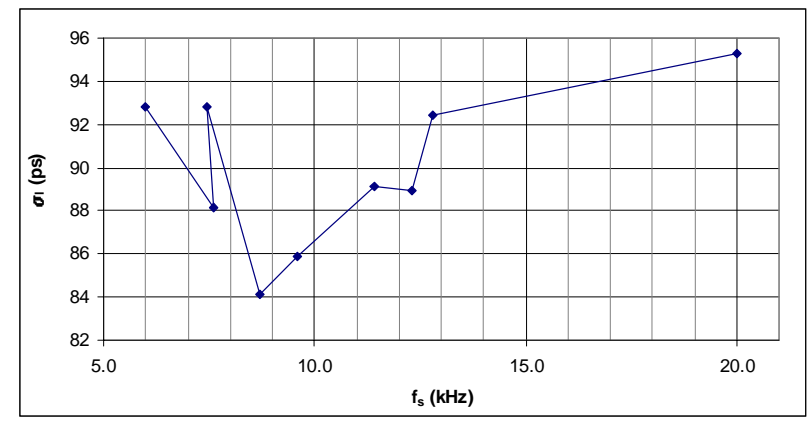

Figure 5: Bunch length (one sigma) as a function of the synchrotron oscillation frequency during migration from the standard mode $\left(f_{\mathrm{s}}=20 \mathrm{kHz}\right)$ to the quasi-isochronous mode $\left(\mathrm{f}_{\mathrm{s}}=6 \mathrm{kHz}\right)$. Initially the measured bunch length and the synchrotron frequency decrease with the reduction of the momentum compaction factor. From the configuration which corresponds to $\mathrm{f}_{\mathrm{s}}=8.5 \mathrm{kHz}$ on, further reduction of the momentum compaction factor still decreases the synchrotron frequency but not the measured bunch length, which starts fluctuating at larger values.

\section{CONCLUSIONS}

We have reduced the momentum compaction factor of the LNLS UVX storage ring by a factor of approximately 13 . The isochronicity parameter $\chi$ has been reduced from 0 to -0.74 . Measurements of bunch length, synchrotron oscillation frequency and orbit displacement have been carried out. Thus far the obtained results were analysed using linear theory only. It is clear, however, that higher order longitudinal terms must be considered. To proceed in the migration route to the QI6 mode we need to consider the effects of the second order momentum compaction factor $\alpha_{2}$ by adding configurations to the migration route where the setting of the sextupoles is considered. The steps in the configurations should be reduced as well.

The experience gained from these initial tests has helped us to determine the limitations of the present measurement system and to prepare an upgrade program to perform future experiments, which includes implementation of the single bunch operation mode and of a single pass bunch length measurement system.

\section{REFERENCES}

[1] H.Hama, S.Takano and G.Isoyama, NIM A329 (1993); P.Tran et al, 1993 PAC; A.Nadji et al, 1994 EPAC; D.Robin et al, Micro Bunches Workshop (1995). 